\title{
Philosophiques
}

\section{La notion de croyance : une approche inscriptionnaliste}

\section{Claude Panaccio}

Volume 15, numéro 1, printemps 1988

URI : https://id.erudit.org/iderudit/027033ar

DOI : https://doi.org/10.7202/027033ar

Aller au sommaire du numéro

Éditeur(s)

Société de philosophie du Québec

ISSN

0316-2923 (imprimé)

1492-1391 (numérique)

Découvrir la revue

Citer cet article

Panaccio, C. (1988). La notion de croyance : une approche inscriptionnaliste. Philosophiques, 15(1), 41-58. https://doi.org/10.7202/027033ar

\section{Résumé de l'article}

On présente ici une interprétation nominaliste des contextes linguistiques indirects comme «A dit que $p$ » et «A croit que $p$ ». L'approche est apparentée à celle de Donald Davidson, mais elle s'en écarte aussi de manière significative. Elle permet de résoudre certaines objections courantes contre l'élimination ontologique des types linguistiques abstraits (celles de George Bealer notamment) ainsi que l'énigme célèbre formulée par Saul Kripke à propos de la notion de croyance. 
PHILOSOPHIQUES, Vol. XV, Numéro 1, Printemps 1988

\title{
LA NOTION DE CROYANCE : UNE APPROCHE INSCRIPTIONNALISTE *
}

\author{
par Claude Panaccio
}

RÉSUMÉ. On présente ici une interprétation nominaliste des contextes linguistiques indirects comme « $A$ dit que $p$ » et « $A$ croit que $p$ ». L'approche est apparentée à celle de Donald Davidson, mais elle s'en écarte aussi de manière significative. Elle permet de résoudre certaines objections courantes contre l'élimination ontologique des types linguistiques abstraits (celles de George Bealer notamment) ainsi que l'énigme célèbre formulée par Saul Kripke à propos de la notion de croyance.

\begin{abstract}
This paper presents a nominalistic interpretation of indirect linguistic contexts such as " $A$ says that $P$ " and " $A$ believes that $\mathrm{p}$ ". The approach has some similarity with that of Donald Davidson, but it also significatively departs from it. It provides solutions to some standard objections against the ontological elimination of abstract linguistic types (those of George Bealer in particular) as well as to the famous puzzle raised by Saul Kripke about the notion of belief.
\end{abstract}

Distinguons soigneusement, à propos des mots, entre les occurrences et les types («tokens» et «types» en anglais). Une occurrence d'une expression linguistique quelconque est spatiotemporellement localisée, elle est liée à un locuteur unique et à un acte d'énonciation singulier. Le type, lui, est plus abstrait : il doit rester identique à lui-même sous les diverses manifestations sonores ou visuelles de ce que nous appelons tout naturellement un même mot (ou une même expression). Dans:

\footnotetext{
* Une première version de ce rexte a été présentée au treizième congrès de la Société de Philosophie du Québec, tenu à Montréal en mai 1986, à l'occasion d'une table ronde sur la notion de croyance avec Daniel Laurier, François Lepage et Michel Seymour. La recherche dont il est issu a été subventionnée par le Conseil de recherches en sciences humaines du Canada et par le Fonds F.C.A.R., auxquels j'exprime ici ma gratitude.
} 
(1) Le chat est un chat, on trouve, par exemple, deux occurrences distinctes d'un même type, le mot «chat». Et si la même phrase (type) est reproduite ailleurs, c'est à d'autres occurrences encore qu'on aura alors affaire.

L'approche inscriptionnaliste des phénomènes de langage part de l'idée qu'il n'existe en réalité que des occurrences. Les types apparaissent dès lors comme des pseudo-entités, des mirages ontologiques. Selon cette conception, quand on dit :

(2) Le mot français «chat » est un nom, on ne parle pas, contrairement aux apparences, d'un objet unique, abstrait par rapport aux occurrences, au-dessus ou en-dessous d'elles ; on ne parle que des occurrences elles-mêmes. Le philosophe américain Wilfrid Sellars, en particulier, a beaucoup insisté sur l'idée qu'une expression comme «le mot chat» est en réalité un terme distributif, comme on en retrouve dans les phrases suivantes :

(3) Le lion est un fauve.

(4) L'être humain est un mammifère.

Les expressions au singulier «le lion» ou «l'être humain » sont utilisées dans de telles phrases au lieu des expressions plurieiles « les lions » ou « les êtres humains », mais elles leur sont à peu près équivalentes. (3) veut dire quelque chose comme:

(5) Les lions sont (normalement) des fauves; et (4) veut dire quelque chose comme:

(6) Les êtres humains sont (normalement) des mammifères. Il en va de même, selon Sellars, de l'énoncé (2), qui se ramène par analyse à :

(7) Les occurrences françaises « chat »(s) sont (normalement) des noms, ce qu'il écrit parfois sous la forme:

(8) Les «chat »s sont des noms ${ }^{1}$.

Cette approche, développée aussi par d'autres auteurs ${ }^{2}$, est d'inspiration résolument nominaliste et naturaliste, voire, le cas

1. Cf. Sellars (1963), (1970), (1974), (1979, chap. 4: «Meaning and ontology 》).

2. Cf. Carnap (1954), (1956), Gochet (1972), Goodman et Quine (1947), Goodman (1977, p. 261 ss.), QUINE (1960, p. 211 ss.), SCHEFFLer (1954), (1979). 
échéant, matérialiste. Elle propose de faire l'économie, dans notre compréhension théorique des phénomènes de langage, de toute une catégorie d'entités abstraites, les types linguistiques, pour nous ramener les deux pieds sur terre aux seuls événements concrets d'énonciation par des locuteurs singuliers réels. A fortiori, elle veut pouvoir se dispenser des propositions ou des concepts qui, dans la sémantique frégéenne, sont censés être "exprimés» par les signes. En évitant ainsi le recours à des entités fantômatiques, elle présente prima facie d'importants avantages d'économie théorique et d'intelligibilité. Souvenons-nous du rasoir d'Occam: il ne faut pas multiplier les entités - il vaudrait mieux dire : les sortes d'entités - sans nécessité.

De sérieuses difficultés, pourtant, la menacent en ce qui a trait à l'analyse des contextes indirects comme ceux qui sont associés à la notion de croyance, les contextes de forme «A croit que $\mathrm{p}$ ». Bealer (1982), par exemple, s'exprime comme si l'inscriptionnalisme, à cause précisément de ces difficultés, était aujourd'hui réfuté ${ }^{3}$. Or je voudrais défendre ici une certaine analyse inscriptionnaliste des contextes indirects. Je me concentrerai d'abord sur le cas des contextes de citation indirecte, de forme «A dit que $p$ », « $A$ affirme que $\mathrm{p}$ ", etc. ; je proposerai à leur sujet une analyse qui, dans la foulée de celle de Davidson (1968), permet de répondre aux deux objections que Bealer trouve décisives contre l'inscriptionnalisme, et $j$ 'indiquerai sur quels points au juste je me sépare de Davidson et pourquoi. J'appliquerai dans un deuxième temps les résultats ainsi obtenus à l'analyse des énoncés qui attribuent des croyances et je tirerai de là une solution toute naturelle à l'énigme formulée par Kripke (1979).

\section{LE DISCOURS INDIRECT}

Développant et modifiant quelque peu la suggestion de Davidson, j'aboutis - provisoirement - à l'interprétation suivante des contextes de citation indirecte :

(9) A affirme que $p$ signifie quelque chose comme:

3. CF. BEALER (1982, p. 28 s.); voir aussi THOMASON (1977). 
(10) A énonce affirmativement et en toute bonne compétence linguistique une occurrence équivalente à : $p$.

Ce n'est encore qu'une approximation certes, et l'on aura cidessous l'occasion de la préciser davantage, mais elle appelle tout de suite un certain nombre de remarques.

Notons d'abord que l'acte illocutoire y est traité comme une modalité (exprimée par un adverbe) de l'acte concret d'énonciation. De la même façon:

(11) A demande si $\mathrm{p}$ deviendrait :

(12) A énonce interrogativement une occurrence équivalente à : $\mathrm{p}$;

et ainsi de suite pour les autres actes illocutoires. Cela indique, me semble-t-il, la voie à suivre pour articuler ensemble l'inscriptionnalisme et la théorie des actes de discours, mais je ne m'y engagerai pas ici plus avant. D'autre part, la clause « en toute bonne compétence linguistique » permet seulement d'éviter pour le moment d'achopper sur le cas où le locuteur dit « il pleut » en voulant dire autre chose, par exemple «le chat est sur le paillasson», par lapsus ou par incompétence linguistique.

L'important pour notre propos est que selon cette analyse, c'est bien une occurrence qui est énoncée plutôt qu'un type ou une proposition. Et le rapporteur caractérise pour le bénéfice de son propre allocutaire l'occurrence produite par le locuteur original en énonçant lui-même surle champ - dans un contexte approprié une nouvelle occurrence sémantiquement équivalente à l'originale. Le «il pleut» de l'énoncé :

(13) Noé dit qu'il pleut est ainsi produit par le rapporteur (et non pas évidemment par Noé) dans un contexte spécial de forme «A dit que __» pour donner à son allocutaire une idée (approximative, du moins) du contenu d'une autre occurrence, produite celle-là par Noé. Cela explique notamment l'usage des expressions indexicales en contextes indirects. Dans :

(14) Georges affirme que je me trompe, « je » dénote en effet le rapporteur et non pas le locuteur original. Cela s'analyse comme suit : 
(15) Georges énonce (...) une occurrence équivalente à : je me trompe (que j'énonce, moi, rapporteur)

Georges, dans ce cas, a pu dire quelque chose comme :

(16) Claude Panaccio se trompe, qui équivaut bel et bien au « je me trompe» qui figure dans (14) comme à celui qui figure dans (15).

Le rapporteur, évidemment, en énonçant une phrase de forme "A dit que $\mathrm{p}$ ", ne prend pas à son propre compte l'acte illocutoire habituellement accompli par l'énonciation de la phrase «p». Lorsque j'énonce (14), par exemple, je n'affirme pas moimême que je me trompe. Il faut considérer qu'un contexte indirect comme "A dit que —— a ceci de spécial qu'il suspend les engagements illocutoires ordinairement associés à l'énonciation de la phrase qui y figure en position de subordonnée. Cette constatation, à la fois banale et très importante, oblige à complexifier quelque peu notre analyse originale. Selon celle-ci, en effet, le foncteur «dit que » doit pouvoir être remplacé par : «produit une occurrence équivalente à ». Or l'occurrence produite par le rapporteur après le «que» n'équivaudra justement pas à celle du locuteur original puisque son énonciation (en contexte indirect) se trouve automatiquement délestée des engagements illocutoires que le locuteur original, lui, a dû contracter. Il faut donc substituer à (10) quelque chose comme :

(17) A énonce (...) une occurrence équivalente à ce que serait normalement: $\mathrm{p}$ (sous-entendu: si je l'énonçais directement et sérieusement).

En d'autres termes, le contexte «A dit que » introduit une situation de jeu scénique. Le rapporteur fait comprendre à son allocutaire qu'il jouera - après le «que » et jusque à la fin de la phrase - un certain rôle illocutoire par lequel, pourtant, il n'entend pas être lui-même lié. Le même phénomène se produit aussi, et de façon encore plus visible, dans le cas de la citation directe : le rapporteur joue alors carrément, pour le bénéfice de l'allocutaire, le rôle du locuteur original. Dans la citation indirecte, le rapporteur conserve, même en jouant, son identité propre et sa situation spatio-temporelle (comme le montre son usage des indexicaux), mais il parvient néanmoins à communiquer à son allocutaire 
le contenu exprimé par le locuteur original en faisant mine de dire lui-même sur le champ quelque chose d'équivalent.

Quant à la relation d'équivalence qui est ici requise, son analyse exacte fait évidemment problème, mais je ne la préciserai pas davantage dans le cadre du présent article. Notons seulement que de toute façon nous disposons pour rapporter en style indirect les paroles d'autrui d'une marge de manœuvre aux limites variables et floues. Si Catherine a dit, par exemple :

(18) De scintillantes goutelettes tombent du firmament, il est admissible dans certains contextes de rapporter plus prosaïquement le contenu de son discours par:

(19) Catherine dit qu'il pleut.

Ce sont apparemment des facteurs d'ordre pragmatique qui déterminent la sorte (ou le degré) d'équivalence qui sera pertinente dans un contexte donné. Sans doute revient-il à la sémantique générale de caractériser de façon abstraite et systématique les différentes relations d'équivalence sémantique possibles entre occurrences linguistiques, et à la pragmatique de spécifier la façon dont le contexte de communication détermine laquelle de ces relations doit être invoquée dans telle situation particulière de compte rendu ou de traduction.

Voyons maintenant comment notre analyse permet de répondre à deux objections souvent adressées à l'inscriptionnalisme et qui sont justement celles que Bealer (1982) reprend à son compte. La première fut originalement adressée à Carnap par Church (1950). Elle conteste qu'une phrase comme :

(20) Sénèque a écrit que l'homme est un animal rationnel puisse être analysée sous la forme de:

(21) Sénèque a écrit dans une certaine langue $L$ une phrase dont la traduction française est «l'homme est un animal rationnel ",

parce que (21) incorpore une référence explicite à une langue donnée, celle dans laquelle la traduction est formulée - ici, c'est le français -, qui ne se trouve pas dans (20). L'énoncé (20), remarque Church, ne pourrait être inféré de (21) que par l'intermédiaire de la prémisse empirique : 
(22) La phrase française «l'homme est un animal rationnel » signifie que l'homme est un animal rationnel.

Pour mieux voir la difficulté, on procède à ce qu'on appelle aujourd'hui le test de traduction de Langford-Church ${ }^{4}$. On traduit par exemple (20) en anglais, ce qui donne:

(23) Seneca wrote that man is a rational animal. Puis on traduit (21), ce qui donne:

(24) Seneca wrote in a certain language $\mathrm{L}$ a sentence which translates in French as "l'homme est un animal rationnel».

Or si on menait directement en anglais l'analyse de (23) selon la méthode utilisée en (21), on obtiendrait :

(25) Seneca wrote in a certain language $\mathrm{L}$ a sentence which translates in English as «man is a rational animal». L'anomalie est que (24) et (25) ne sont pas synonymes : il est fait référence dans l'une au français et dans l'autre à l'anglais. Et de toute façon, la phrase originale (20) ne renvoyait, elle, ni au français ni à l'anglais. Cela révèle de façon décisive un défaut majeur de la méthode d'analyse utilisée en (21).

Puisque la difficulté tient à la référence explicite à une langue* donnée, on doit se demander si l'analyse inscriptionnaliste ne pourrait pas tout simplement se passer de cet indésirable renvoi. Non, répond Church, parce qu'il peut arriver qu'une même séquence de sons (ou de marques écrites) possède dans deux langues différentes deux significations toutes différentes. Le cas est rare certes, mais il est toujours empiriquement possible et j'ai, à ce propos, concocté en adaptant une vieille blague de collégien, l'exemple suivant. Supposons que le capitaine Haddock énonce en anglais la phrase suivante:

4. ChuRCH (1950) attribue en effet l'idée originale d'un tel test à C.H. Langford. Le principe en est qu'une bonne analyse d'une certaine catégorie d'énoncés doit être telle que si elle est menée dans une langue $L_{1}$ à propos d'un énoncé $E_{1}$ de la même langue, alors la traduction de cette analyse dans une autre langue $\mathrm{L}_{2}$ doit être strictement synonyme d'une nouvelle analyse, menée selon la même méthode mais directement dans $L_{2}$, d'un énoncé $E_{2}$ qui est, lui, la traduction de $E_{1}$ dans $L_{2}$, cela en vertu de la transitivité de la synonymie, qui doit être préservée aussi bien par l'analyse que par la traduction. 
(26) Nestor quickly brought the rhum cakes.

On est alors autorisé à dire en français oral:

(27) Haddock dit que Nestor apporta vite les gâteaux au rhum,

ce qui, dans une analyse de type carnapien qui serait délestée de la référence explicite à la langue de la traduction (ici le français), deviendrait :

(28) Haddock dit quelque chose qui équivaut à la phrase (orale) : «Nestor apporta vite les gâteaux au rhum».

Or si on ne précise pas la langue à laquelle appartient la phrase mentionnée entre guillemets dans (28), il pourrait évidemment s'agir de la phrase française :

(29) Nestor apporta vite les gâteaux au rhum, mais il pourrait tout aussi bien s'agir de la phrase latine:

(30) Nestor apportavit legato aurum, qui se traduit en français par:

(31) Nestor apporta de l'or au député. Notre analyse (28) serait alors - tant qu'on en reste au niveau oral - incapable de différencier (27), qui, dans le cas considéré, est vraie, de :

(32) Haddock dit que Nestor apporta de l'or au député, qui, elle, est complètement fausse. Il faut donc apparemment revenir à une analyse comme :

(33) Haddock dit quelque chose qui équivaut à la phrase (orale) française: «Nestor apporta vite les gâteaux au rhum».

Mais dès qu'on réintroduit ainsi la référence explicite au français, on retombe sous le coup de l'objection de Church: la phrase originale (27) ne contenait aucune référence au français (comme le montre le test de traduction).

L'analyse que je défends ici - comme celle de Davidson d'ailleurs - permet de régler cette difficulté de la façon suivante : puisque l'occurrence de la subordonnée « $\mathrm{p}$ » est considérée comme étant produite sur le champ par le rapporteur, il sera pragmatiquement présupposé qu'elle est formulée dans la langue que parle celui-ci, le français dans le cas de (20) et l'anglais dans le cas de 
(23). La référence à cette langue n'a donc nul besoin d'être rendue explicite dans l'analyse. Après tout, la référence au français ne fait pas partie du contenu sémantique de chacune des phrases du français. L'équivocité interlinguistique - comme celle qui affecte les phrases orales représentées par (29) et (30) - est néanmoins évitée si les interlocuteurs savent déjà quelle est la langue que parle le rapporteur, ce qui est normalement présupposé en contexte de communication. L'important ici est que la subordonnée " $p$ » n'est pas une description de phrase, comme chez Carnap et chez Quine ', ni un nom de phrase, ni, comme dans Scheffler (1954), un prédicat général applicable à une pluralité d'autres occurrences. Il s'agit plutôt d'une occurrence produite par le rapporteur dans sa propre langue, mais dans un contexte spécial où, conventionnellement, les engagements illocutoires habituels sont suspendus. L'analyse de l'exemple (20) sera rendue par:

(34) Sénèque a écrit (...) quelque chose qui équivaut à ce que serait normalement : l'homme est un animal rationnel.

Et l'on peut vérifier que cette méthode d'analyse réussit facilement le test de Langford-Church. La traduction anglaise de (34) en effet coïncidera exactement avec l'analyse menée directement en anglais (selon la même méthode) de la phrase (23), qui est, elle, la traduction anglaise de (20). La langue parlée par le rapporteur étant supposée identifiée par ailleurs (sans quoi il n'y aurait pas de communication), on échappe en même temps aux équivocités du genre de celle qui affectait l'analyse (28).

Cela règle du même coup un autre problème grave, que rencontre notamment l'inscriptionnalisme de Scheffler (1954). Celui-ci considère le « $p$ » des énoncés de forme « $A$ dit que $p$ » comme étant en réalité un prédicat métalinguistique. Il analyse comme suit l'exemple (20):

5. Quine (1960, p. 143) suggère en effet qu'un énoncé qui figure dans le contexte d'une citation peut être vu comme une description métalinguistique de l'énoncé original (qui en indiquerait, par exemple, l'épellation) er il envisage de recourir à la même approche pour l'analyse des contextes indirects (p. 212). Il faut noter cependant qu'il se déclare finalement insatisfait de cette solution. Il recommande pour les fins du langage discipliné qu'il voudrait voir adopter par la science l'abandon pur et simple des foncreurs d'attirude propositionnelle (p. 216 ss.). 
(35) Sénèque a écrit quelque chose qui est un «l" - homme - est - un - animal - rationnel», où l'expression «est un 'l' - homme - est - un - animal rationnel" » est un prédicat général primitif qui s'applique aux occurrences de n'importe quelle langue qui équivalent à «l'homme est un animal rationnel ». Scheffler semble ainsi obligé d'admettre pour chaque type linguistique un prédicat métalinguistique primitif correspondant. Or comme nos langues naturelles peuvent en principe incorporer une infinité de types linguistiques différents - en particulier une infinité de phrases différentes - , on aura besoin dans cette approche d'une infinité de prédicats métalinguistiques primitifs. Mais une langue comprenant une infinité de prédicats primitifs ne pourrait pas être apprise par des locuteurs finis (comme nous le sommes). L'inscriptionnalisme de Scheffler contreviendrait ainsi à une contrainte fort plausible proposée par Davidson (1965) : la langue dans laquelle une analyse sémantique est développée doit pouvoir être apprise par des locuteurs aux capacités finies ${ }^{6}$. Notre propre analyse - comme celle de Davidson toujours - évite cette difficulté puisqu'elle ne recourt justement pas à des prédicats métalinguistiques spéciaux, mais à des occurrences de phrases du langage-objet lui-même, lesquelles sont apprises de la même façon que n'importe quelle autre phrase du langage-objet, à partir d'un vocabulaire fini et d'un nombre fini de règles de composition.

Jusqu'ici, mon analyse des contextes indirects semble assez proche de celle de Davidson (1968) et j'ai d'ailleurs souligné au passage - sans fournir d'explication - que cette dernière permet également de répondre aux deux objections que je viens de discuter. L'approche de Davidson, cependant, a elle-même suscité, depuis qu'elle a été proposée, un certain nombre de critiques qui en ont fait apparaître l'inconvénient central 7 . Il me faut donc préciser la différence exacte qu'il y a entre mon analyse et la sienne et montrer ainsi comment on peut conserver les avantages de la

6. Cette critique est formulée notamment par $\operatorname{BealeR}(1982$, p. 28).

7. CF. BLACKBURN (1975), Foster (1976), LoAR (1976), BALDWIN (1982). On trouvera des réponses à certaines de ces critiques dans DAvidson (1976) et SMITH (1976). Je ne résumerai pas ici l'ensemble de la discussion pour me concentrer plutôt sur l'objection la plus importante, celle à laquelle Davidson ne semble pas en mesure de répondre sans modifier sa théorie. 
théorie davidsonnienne tout en échappant à la difficulté principale sur laquelle elle achoppe. Cela permettra d'approfondir encore et de façon particulièrement révélatrice - la portée de la théorie que je défends.

Selon Davidson, la phrase (20) citée ci-dessus s'analyserait comme suit :

(36) Sénèque a écrit quelque chose qui équivaut à ceci: l'homme est un animal rationnel.

L'idée de base est que le rapporteur énonce en fait deux phrases distinctes et juxtaposées - séparées dans (36) par les deux points - et que dans l'une de ces phrases (la première), il réfère à l'autre. Le « que » de «A croit que $\mathrm{p}$ » cacherait à la fois un démonstratif et une ponctuation forte capable de séparer des phrases distinctes. C'est pourquoi cette approche est souvent appelée «l'analyse parataxique des contextes indirects ", la parataxe étant en grammaire une construction par simple juxtaposition. Comme la deuxième de ces phrases est énoncée par le rapporteur au sein de son propre discours et, il faut le présupposer, dans sa propre langue, elle ne constitue en rien une description métalinguistique, ce qui, comme on l'a vu, permet de contrer certaines objections classiques.

Le problème crucial, cependant, tient à l'introduction dans la première phrase d'une référence indexicale explicite à la seconde 8 . Cela soulève le même genre de difficultés que ce que l'on rencontrait déjà dans l'analyse (28) à propos de la référence explicite à une langue. Il est en effet difficile de croire que l'énoncé (20) implique de par son contenu sémantique même non seulement l'existence d'une énonciation du locuteur original (dans ce cas, Sénèque), mais aussi l'existence d'une phrase énoncée par le rapporteur lui-même, celle à laquelle le démonstratif est censé renvoyer. De nouveau, le test de traduction s'avère révélateur. La traduction anglaise littérale de (36) devrait conserver la référence indexicale à la séquence française «l'homme est un animal rationnel » qui figure dans (36), alors qu'une analyse de (23) directement menée en anglais selon la méthode de Davidson devrait, quant à elle, contenir une référence indexicale à la phrase anglaise «man is a rational animal» qui y figurera.

8. Je remercie Daniel Laurier d'avoir ${ }_{3}$ au moment de la table ronde dont le présent article est issu, judicieusement attiré mon attention sur ce point précis. 
La solution, me semble-t-il, est de renoncer dans notre analyse à l'idée de construction parataxique et d'éliminer par le fait même la source du problème, à savoir la référence indexicale explicite à une phrase produite par le rapporteur. Il faut considérer que dans l'analyse (34), qui est celle que je propose, les deux points ne constituent pas une ponctuation forte séparant deux phrases distinctes, mais une conjonction de subordination d'une nature particulière, capable - conventionnellement - de provoquer un contexte syntaxique spécial qui suspend les engagements illocutoires habituellement liés à l'énonciation de la phrase qui y figure en position de subordonnée, et d'introduire du même coup le jeu scénique auquel le rapporteur va maintenant se livrer. Le « $\mathrm{p}$ » de "A croit que $\mathrm{p}$ » fait ainsi bel et bien partie de la même phrase que le reste à titre de subordonnée, et l'on n'a donc nul besoin d'y référer explicitement. Si l'on veut rendre compte des contextes indirects, c'est notre conception de la syntaxe des langues naturelles qu'il nous faut élargir pour y inclure justement cette sorte particulière de subordonnée ou, plus généralement, cette façon particulière d'imbriquer l'énonciation d'une occurrence linguistique dans une autre.

\section{LA NOTION DE CROYANCE}

Sur la base de cette analyse du discours indirect, on peut maintenant introduire une certaine notion de croyance qui, à bien des égards, rejoint celle du langage ordinaire :

(37) A croit que $\mathrm{p}=\mathrm{df} \mathrm{A}$ est disposé à affirmer que $\mathrm{p}$ (dans certaines circonstances qui restent à déterminer) 9 .

Cette définition stipulative sommaire appelle d'emblée deux remarques. D'abord, une telle notion de croyance ne s'applique qu'à des agents linguistiques : les animaux n'ont pas de croyance en ce senslà. Si on veut leur en attribuer, on peut élargir la notion que je viens d'introduire, en posant par exemple quelque chose comme :

(38) A croit que $\mathrm{p}=\operatorname{df} \mathrm{A}$ a certaines dispositions souvent associées à la disposition à affirmer que $\mathrm{p}$.

9. J'ai déjà utilisé cette définition dans ma reconstruction de la problématique de l'analyse des idéologies; cf. PANACCIO (1984, p. 46). 
Mais je n'entrerai pas ici davantage dans cette discussion, par ailleurs fort intéressante, et je m'en tiendrai pour l'instant à la notion restreinte de croyance comme disposition à affirmer ${ }^{10}$.

Deuxièmement, la disposition en question devrait être caractérisée de manière plus précise comme étant une disposition d'une sorte bien spéciale, susceptible de se manifester de façon privilégiée lorsque le locuteur entreprend de verbaliser son avis sincère sur cela qui est en question dans l'énoncé " $\mathrm{p}{ }^{11}$. Quant à savoir comment de telles circonstances peuvent être empiriquement identifiées, c'est là une question épistémologique cruciale certes, mais qui, de nouveau, outrepasse les limites du présent article.

Il reste à savoir maintenant comment notre analyse inscriptionnaliste permet de résoudre l'énigme de Kripke (1979). Pierre, un français, affirme sincèrement à plusieurs reprises: «Londres est jolie». Installé en Angleterre et devenu anglophone par contact direct avec ses nouveaux voisins, il affirme aussi sincèrement, et sans pour autant renier ses convictions antérieures : «London is not pretty ». C'est qu'il ignore que la ville qu'il connaît maintenant sous le nom anglais de «London » est celle-là même qu'en français on appelle «Londres». Pierre, donc, croit-il que Londres est jolie ou que Londres n'est pas jolie? ${ }^{12}$ Réponse : il croit l'un et l'autre. Si l'on combine, en effet, nos analyses (10), (17) et (37), on obtient :

(39) A croit que $\mathrm{p}=\mathrm{df} \mathrm{A}$ est disposé à énoncer affirmativement et en toute bonne compétence linguistique une occurrence équivalente à ce que serait normalement: $\mathrm{p}$.

10. GOCHET (1972), par exemple, accorde beaucoup d'importance à l'idée qu'une analyse adéquate doit rendre compte de l'attribution de croyances aux animaux dénués de langage. Je ne cherche pas ici cependant à me conformer à tout prix à l'usage ordinaire dans toute sa généralité. En adoptant pour les fins de la présente discussion une définition plus restrictive, il me suffira de retrouver les principales caractéristiques de la notion habituelle de croyance qui s'applique aux agents linguistiques.

11. Qu'on pense ici à l'idée de réflexion spontanée à haute voix («thinking-out-loud») présentée comme cruciale par SELLARS (1974, p. 419 ss., et 1979, p. 75 ss.) pour la compréhension philosophique des phénomènes de pensée. Il n'est pas dit en tout cas que dans une reconstruction conceptuelle ordonnée, la notion de verbalisation sincère doive nécessairement présupposer celle de croyance.

12. On trouvera une présentation beaucoup plus détaillée de cette énigme dans l'article de Michel Seymour, "Les énoncés de croyance et l'énigme de Kripke», publié dans la présente livraison. 
Or on a bel et bien dans le récit de Kripke l'état de choses suivant :

(40) Pierre est disposé à énoncer (...) certaines occurrences équivalentes à (...): Londres est jolie.

D'où l'on peut conclure :

(41) Pierre croit que Londres est jolie.

Et l'on a également cet autre état de choses :

(42) Pierre est disposé à énoncer (...) certaines occurrences équivalentes à (...): Londres n'est pas jolie.

Il s'agit alors des occurrences anglaises de «London is not pretty ». De (39) et (42), on peut alors conclure :

(43) Pierre croit que Londres n'est pas jolie. Notons que puisque Pierre n'est prêt à affirmer aucune occurrence équivalente à : Londres est et n'est pas jolie à la fois, il s'ensuit :

(44) Pierre ne croit pas que Londres est et n'est pas jolie à la fois.

Le véritable problème posé par l'énigme de Kripke est que nous étions acculés à une contradiction logique puisqu'il nous fallait apparemment admettre à la fois :

(45) Pierre croit que Londres est jolie et :

(46) Pierre ne croit pas que Londres est jolie, ce qui constituerait une contradiction de notre part. La solution inscriptionnaliste montre qu'il y a bel et bien contradiction dans les croyances de Pierre - bien qu'il ne soit pas, à cause des circonstances très particulières, en mesure de s'en apercevoir par un raisonnement purement logique -, mais elle ne nous conduit pas nous-mêmes à une contradiction parce que, contrairement à ce que présuppose Kripke, nous pouvons refuser le schéma d'inférence suivant :

(47) A croit que p; donc: A ne croit pas que non-p.

C'est ce schéma d'inférence qui, chez Kripke, conduisait à une contradiction. Or nous pouvons le refuser parce que - et c'est ici qu'est le secret de la solution - notre analyse inscriptionnaliste recourt à une quantification sur les occurrences: A croit que $\mathrm{p}$ si et seulement si A est disposé à affirmer certaines occurrentes équivalentes à ce que serait normalement: p. À partir du moment où 
nous substituons la pluralité des occurrences à l'unicité de la phrase ou de la proposition, cette quantification s'impose tout naturellement et c'est elle, justement, qui règle la difficulté :

(48) A ne croit pas que non-p s'analysera dès lors comme :

(49) A n'est disposé à affirmer aucune occurrence équivalente à (...) : non-p, ce qui, bien sûr, ne découle pas logiquement de :

(50) A est disposé à affirmer certaines occurrences équivalentes à $(\ldots)$ : p.

De même, être disposé à refuser d'affirmer que $\mathrm{p}$ ne revient pas non plus à croire que non-p. Certes, il reste empiriquement vrai que, dans la plupart des cas, si $A$ croit que $p, A$ ne croit pas que non$\mathrm{p}$, mais il n'y a pas là d'implication logiquement valide.

Certains sans doute s'attristeront de ce résultat qu'ils interpréteront comme une invitation à renoncer purement et simplement à la logique épistémique tout entière. Ils invoqueront une certaine intuition logique qui nous porte spontanément, diront-ils, à établir une relation qui n'est pas seulement empirique entre « $A$ croit que $p$ » et «A ne croit pas que non- $p$ ». Et c'est là une réaction tout à fait saine, mais qui est seulement un peu déplacée. L'intuition en question s'explique, à mon sens, par le caractère logiquement valide du schéma d'inférence suivant :

(51) A croit que $\mathrm{p}$; donc, si les croyances de A sont cohérentes, alors $A$ ne croit pas que non-p.

À partir de quoi, si l'on considère que les agents sont socialement engagés à la cohérence logique, on peut vraisemblablement admettre aussi cet autre schéma d'inférence :

(52) A croit que $p$; donc, $A$ est engagé à ne pas croire que non-p

Mais dans l'historiette de Kripke, les croyances de Pierre ne sont justement pas cohérentes.

Il ne faut par ailleurs abandonner ni l'un ni l'autre des deux principes auxquels Kripke attribue la responsabilité conjointe de son paradoxe, le principe de déguillemetisation (qui est, du reste, impliqué par notre analyse (37)) : 
(53) Si $A$ affirme sincèrement " $\mathrm{p}$ ", alors $A$ croit que $p$, et le principe de traduction (que $j$ 'ai aussi admis tout au long du présent article, notamment dans l'utilisation que j'ai faite du test de Langford-Church):

(54) Si «q" est une bonne traduction de " $p$ », alors « $p$ » et «q» ont la même valeur de vérité.

Ce qu'il faut abandonner, c'est plutôt le principe (47) ci-dessus, qu'on appelle parfois le principe de rationalité et qui est, celui-là, présupposé par Kripke sans discussion. L'analyse inscriptionnaliste proposée ici montre exactement pourquoi ce principe doit être abandonné. La solution de l'énigme n'est pas ici atteinte par un ajustement ad boc, elle découle naturellement d'une position inscriptionnaliste à laquelle on aboutit pour des raisons tout à fait indépendantes.

Département de philosophie,

Université du Québec à Trois-Rivières.

\section{RÉFÉRENCES BIBLIOGRAPHIQUES DES TRAVAUX CITÉS}

BALDWIN, T. (1982), "Prior and Davidson on indirect speech », Philosophical Studies, 42, PP. 255-282.

BEALER, G. (1982), Quality and Concept, Oxford, Clarendon Press.

BLACKBURN, S. (1975), «The identity of propositions », in Meaning, Reference and Necessity, sous la dir. de S. Blackburn, Cambridge, Cambridge U.P.

CARNAP, R. (1954), "On belief-sentences. Reply to Alonzo Church", in Philosophy and Analysis, sous la dir. de M. Macdonald, Oxford, Pp. 128-131 (repris dans Carnap, 1956, pp. 230-232).

(1956), Meaning and Necessity, $2^{\text {nd }}$ ed., Chicago, The Univ. of Chicago

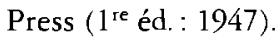

CHURCH, A. (1950), “On Carnap's analysis of statements of assertion and belief », Analysis, 10, pp. 97-99.

DAVIDSON, D. (1965), «Theories of meaning and learnable languages», in Proceedings of the 1964 Congress for Logic, Methodology, and the Philosophy of Science, sous la dir. de Y. Bar-Hillel, Amsterdam, North-Holland (repris dans Davidson, 1984, pp. 3-15). 
(1968), "On saying that", Syntbese, 19, pp. 130-146 (repris dans Words and Objections, sous la dir. de D. Davidson et $\mathrm{H}$. Hintikka, Dordrecht, Reidel, 1969, pp. 158-174; et dans Davidson, 1984, pp. 93-108).

(1976), «Reply to Foster », in Evans et McDowell (1976), pp. 33-41 (repris dans Davidson, 1984, pp. 171-179) Press.

(1984), Inquiries into Truth and Interpretation, Oxford, Clarendon

EVANS, G. et MCDOWELL, J. (1976), Truth and Meaning, Oxford, Clarendon Press.

FOSTER, J.A. (1976), «Meaning and truth theory», in Evans et McDowell (1976), pp. 1-32.

GOCHET, P. (1972), Esquisse d'une théorie nominaliste de la proposition, Paris, Armand Colin.

GOODMAN, N. (1972), Problems and Projects, Indianapolis, Ind., BobbsMerrill.

(1977), The Structure of Appearance, Dordrecht, Reidel (1 $1^{\text {re }}$ éd. : 1951).

GOODMAN, N. et QUINE, W.V.O. (1947), “Steps toward a constructive nominalism ", Journal of Symbolic Logic, 12, pp. 105-122 (repris dans Goodman, 1972, pp. 173-198).

KRIPKE, S. (1979), “A puzzle about belief », in Meaning and Use, sous la dir. de A. Margalit, Dordrecht, Reidel, pp. 239-283.

LOAR, B. (1976), «Two theories of meaning», in Evans et McDowell (1976), Pp. $138-161$.

PANACCIO, C. (1984), “Problématique de l'analyse des idéologies », in L'idéologie et les stratégies de la raison, sous la dir. de C. Savary et C. Panaccio, Montréal, Hurtubise HMH, pp. 35-65.

QUINE, W.V.O. (1960), Word and Object, Cambridge, Mass., The M.I.T. Press.

SCHEFFLER, I. (1954), "An inscriptional approach to indirect quotation", Analysis, 14, PP. 83-90.

(1979), Beyond the Letter, London, Routledge and Kegan Paul.

SELLARS, W. (1963), “Abstract entities », Review of Metaphysics, 16, PP. 627-671 (repris dans Pbilosopbical Perspectives, Springfield, Ill., Charles C. Thomas, pp. 229-269).

(1970), «Toward a theory of the categories », in Experience and Theory, sous la dir. de L. Foster et J.W. Swanson, Boston, Univ. of Massachussets Press, pp. 55-78.

(1974), «Meaning as functional classification», Syntbese, 27, pp. 417-437.

(1979), Naturalism and Ontology, Reseda, Cal., Ridgeview.

SMITH, P. (1976), «Blackburn on saying that», Philosopbical Studies, 30, pp. $424-426$. 
THOMASON, R.H. (1977), «Indirect discourse is not quotational », The Monist, 60 , pp. $340-354$. 